\title{
Activity-Dependent Bulk Endocytosis and Clathrin-Dependent Endocytosis Replenish Specific Synaptic Vesicle Pools in Central Nerve Terminals
}

\author{
Giselle Cheung, Orla J. Jupp, and Michael A. Cousin \\ Membrane Biology Group, Centre for Integrative Physiology, University of Edinburgh, Edinburgh EH8 9XD, United Kingdom
}

\begin{abstract}
Multiple synaptic vesicle (SV) retrieval modes exist in central nerve terminals to maintain a continual supply of SVs for neurotransmission. Two such modes are clathrin-mediated endocytosis (CME), which is dominant during mild neuronal activity, and activitydependent bulk endocytosis (ADBE), which is dominant during intense neuronal activity. However, little is known about how activation of these SV retrieval modes impact the replenishment of the total SV recycling pool and the pools that reside within it, the readily releasable pool (RRP) and reserve pool. To address this question, we examined the replenishment of all three SV pools by triggering these SV retrieval modes during both high- and low-intensity stimulation of primary rat neuronal cultures. SVs generated by CME and ADBE were differentially labeled using the dyes FM1-43 and FM2-10, and their replenishment of specific SV pools was quantified using stimulation protocols that selectively depleted each pool. Our studies indicate that while the RRP was replenished by CME-generated SVs, ADBE provided additional SVs to increase the capacity of the reserve pool. Morphological analysis of the uptake of the fluid phase marker horseradish peroxidase corroborated these findings. The differential replenishment of specific SV pools by independent SV retrieval modes illustrates how previously experienced neuronal activity impacts the capability of central nerve terminals to respond to future stimuli.
\end{abstract}

\section{Introduction}

Central nerve terminals have a limited supply of synaptic vesicles (SVs), which must be quickly and reliably recycled to maintain neurotransmitter release. This is particularly important during elevated neuronal activity, where multiple SVs fuse with the plasma membrane over a short period of time. At least two different SV retrieval modes are triggered during intense neuronal activity. First, clathrin-mediated endocytosis (CME) retrieves single SVs directly from the plasma membrane (Ungewickell and Hinrichsen, 2007; Doherty and McMahon, 2009). CME is the dominant SV retrieval mode during periods of low activity (Granseth et al., 2006; Zhu et al., 2009); however, it has a limited capacity (Sankaranarayanan and Ryan, 2000). This means it cannot cope with increased endocytic demand during high-intensity stimulation (Clayton et al., 2008). During elevated neuronal activity, a second SV retrieval mode is triggered, called activitydependent bulk endocytosis (ADBE). ADBE is a fast, highcapacity SV retrieval mode that invaginates large regions of plasma membrane, creating bulk endosomes from which SVs can bud (Clayton and Cousin, 2009).

Received Jan. 19, 2010; revised April 22, 2010; accepted April 30, 2010.

This work was supported by grants from The Wellcome Trust (084277) and The Cunningham Trust (0015). Dr. Alan Prescott and John James (both University of Dundee) are thanked for excellent technical assistance.

0. J. Jupp's present address: Biomedical Research Centre, School of Biological Sciences, University of East Anglia, Norwich NR4 7TJ, UK.

Correspondence should be addressed to Mike Cousin, Membrane Biology Group, Centre for Integrative Physiology, George Square, University of Edinburgh, Edinburgh EH8 9XD, Scotland, UK. E-mail: m.cousin@ed.ac.uk.

DOI:10.1523/JNEUROSCI.0293-10.2010

Copyright $\odot 2010$ the authors $\quad 0270-6474 / 10 / 308151-11 \$ 15.00 / 0$
SVs that are available for exocytosis in central nerve terminals are commonly referred to as the recycling pool (Südhof, 2000; Rizzoli and Betz, 2005). This pool can be further subdivided into the readily releasable pool (RRP) and the reserve pool. The RRP contains SVs that are immediately available for fusion, whereas the reserve pool contributes SVs for fusion during periods of intense neuronal activity (Südhof, 2000; Rizzoli and Betz, 2005). However, it is still unclear how these SV pools are themselves replenished by different endocytic mechanisms. During mild stimulation, SVs are preferentially recycled to repopulate the RRP in a process called "reuse" (Pyle et al., 2000; Kavalali, 2006). This "reuse" route also refills the RRP during high-intensity stimulation (Sara et al., 2002), suggesting that this mechanism occurs across a wide activity range. SVs generated by ADBE are not immediately available for release in amphibian neuromuscular junctions, indicating that they may repopulate the reserve SV pool instead (Richards et al., 2000; Richards et al., 2004). In agreement, ADBE-generated SVs support a sustained component of SV fusion evoked by intense neuronal activity in central nerve terminals (Evans and Cousin, 2007; Clayton et al., 2009).

We investigated the replenishment of the RRP and reserve pools by both CME and ADBE during low- and high-intensity stimulation. SVs generated by these two SV retrieval modes were visualized using the styryl dyes FM1-43 and FM2-10, since these probes differentially label CME and ADBE (Richards et al., 2000; Virmani et al., 2003; Richards et al., 2004; Evans and Cousin, 2007; Clayton and Cousin, 2008). We corroborated our findings via parallel morphological studies with the fluid phase marker horseradish peroxidase (HRP). We found that the RRP was selec- 


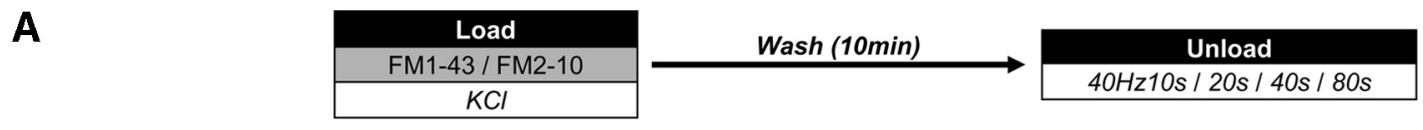

B

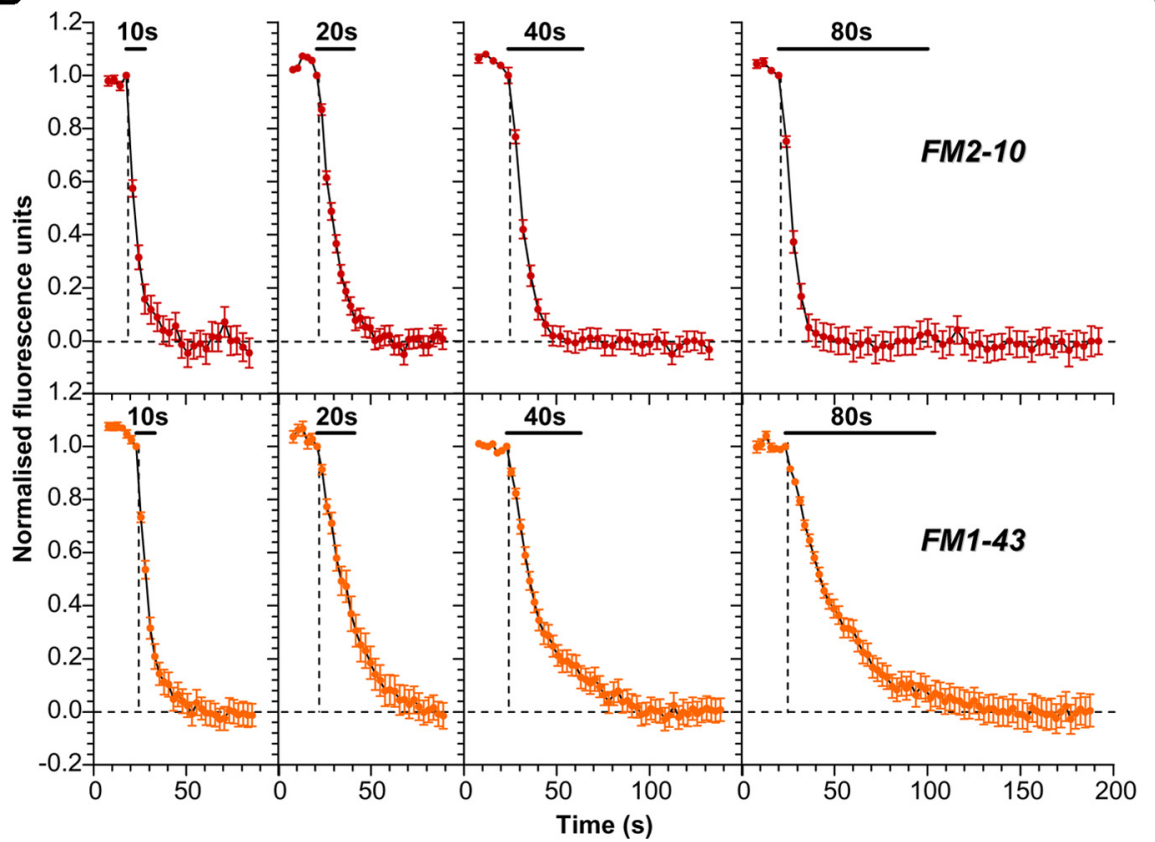

C
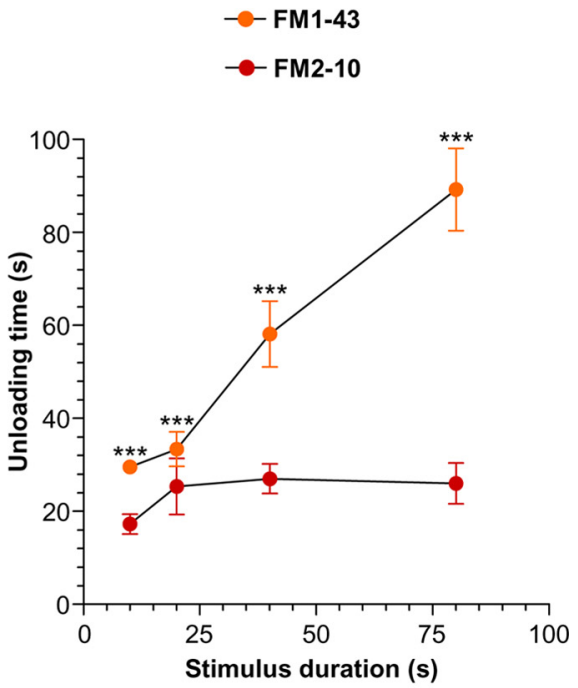

Figure 1. ADBE provides additional SVs for the total SV recycling pool. $A$, Cultures were loaded with either $10 \mu \mathrm{m}$ FM1-43 or $100 \mu \mathrm{m} \mathrm{FM2-10} \mathrm{in} \mathrm{the} \mathrm{presence} \mathrm{of} \mathrm{elevated} \mathrm{KCI} \mathrm{(50} \mathrm{mM)} \mathrm{for} 2$ min. Dyes were washed away immediately after stimulation, and cultures were left to rest for $10 \mathrm{~min}$. Dye unloading was stimulated by trains of action potentials (either $10 \mathrm{~s}, 20 \mathrm{~s}, 40 \mathrm{~s}$, or $80 \mathrm{~s}$ ) at $40 \mathrm{~Hz}$. $B$, Representative traces of the time course of dye unloading in response to action potentials are displayed (FM2-10, red symbols, top; FM1-43, orange symbols, bottom). The duration of unloading stimulation is represented by bars. Data are normalized between 1 and 0 in all instances, where $1=$ start of stimulation and $0=$ cessation of unloading (indicated by dotted lines). $\boldsymbol{C}$, The mean unloading time for either dye is plotted against the stimulus duration (FM2-10, red symbols; FM1-43, orange symbols). In all experiments, $n=4$ (except $40 \mathrm{~Hz} 10$ s FM1-43 and $40 \mathrm{~Hz} 80 \mathrm{~s}$ FM1- 43 , $n=3$ ); error bars represent \pm SEM; ${ }^{* *} p<0.001$, two-way ANOVA.

tively replenished by SV generated by CME, whereas ADBE generates additional SVs to increase reserve pool capacity. These studies highlight the fact that previous patterns of activity are key in shaping the capability of central nerve terminals to respond to future trains of stimuli.

\section{Materials and Methods}

Materials. FM2-10, FM1-43, penicillin/streptomycin, phosphate-buffered salts, fetal calf serum, and Minimal Essential Medium were obtained from Invitrogen. Glutaraldehyde and osmium tetroxide were from Agar Scientific. Cyclosporin A was from Merck. All other reagents were from Sigma. shRNA against syndapin I was designed using the PSUPER vector system (pSUPER neo-GFP, OligoEngine), using the following oligonucleotides: Oligo A1, AGAAGAAACTTGTGGATAA; Oligo B1, AGAAGCCCTGGGCTAAGAA. The pSUPER vector was engineered to express mCerulean by removing GFP with the enzymes AgeI and BsrGI and replacing with predigested mCerulean.

Primary culture and transfections of cerebellar granule neurons. Primary cultures of cerebellar granule neurons were prepared from cerebella of 7-d-old Sprague Dawley rat pups (both male and female) according to previously described protocols (Tan et al., 2003). Cultures were grown for 8-10 d in vitro before use in experiments. Transfections were performed using calcium phosphate precipitation (Tan et al., 2003). Experiments using transfected neurons were performed $72 \mathrm{~h}$ after transfection.

Fluorescence imaging of SV turnover. Cells were removed from culture medium and left to repolarize for $10 \mathrm{~min}$ in incubation medium [in mM: $170 \mathrm{NaCl}, 3.5 \mathrm{KCl}, 0.4 \mathrm{KH}_{2} \mathrm{PO}_{4}, 20$ TES (N-tris[hydroxyl-methyl]methyl-2-aminoethane-sulfonic acid), $5 \mathrm{NaHCO}_{3}, 5$ glucose, 1.2 $\mathrm{Na}_{2} \mathrm{SO}_{4}, 1.2 \mathrm{MgCl}_{2}$, and $1.3 \mathrm{CaCl}_{2}$; at $\mathrm{pH}$ 7.4]. Cultures were then mounted in a Warner imaging chamber (RC-21BRFS). Invaginating membrane was loaded with either FM2-10 $(100 \mu \mathrm{M})$ or FM1-43 (10 $\mu \mathrm{M})$ by evoking SV turnover using either electrical field stimulation $(100 \mathrm{~mA}$, $1 \mathrm{~ms}$ pulse width, delivered using platinum wires embedded in the imaging chamber) or elevated $\mathrm{KCl}(50 \mathrm{~mm} \mathrm{KCl}$, with $50 \mathrm{~mm} \mathrm{NaCl}$ removed to maintain osmolarity). Dye was washed from the cultures immediately after stimulation terminated. Accumulated dye was unloaded from nerve terminals after either $10 \mathrm{~min}$ (standard unload) or after $2 \mathrm{~min}$ (immediate unload) using either electrical stimulations or elevated $\mathrm{KCl}$ medium as described in figure legends.

In some cases, S1/S2 experiments were performed in which cultures underwent two rounds of dye loading and unloading separated by a 20 min rest period. This approach allowed a direct comparison of the total number of SVs turned over in both the S1 and S2 loads. Individual loading and unloading protocols for these experiments are detailed in Results.

Dye loading and unloading of untransfected cultures was visualized using a Nikon Diaphot-TMD epifluorescence microscope at $480 \mathrm{~nm}$ excitation and $>510 \mathrm{~nm}$ emission with a $20 \times$ air objective. Experiments using transfected cultures were performed using a Zeiss TV-100 epifluorescence microscope and a $40 \times$ oil objective. Transfected neurons were visualized at $430 \mathrm{~nm}$ excitation, whereas FM1-43 was visualized at 500 $\mathrm{nm}$ (both $>525 \mathrm{~nm}$ emission). Fluorescent images were captured at $4 \mathrm{~s}$ intervals using a Hamamatsu Orca-ER CCD digital camera and processed using offline imaging software (Simple PCI, Compix Imaging Systems). Regions of interest of identical size were placed over randomly selected nerve terminals, and the total fluorescence intensity of these regions was monitored over time. Only nerve terminals that displayed stimulation-dependent dye loading and unloading were used for analysis. For each experiment, fluorescent time courses from $\sim 90$ nerve terminals were normalized to the same initial fluorescence level. The average fluorescence drop of each unloading step (stimuli to unload either the RRP or reserve pool) was expressed as a percentage of the total fluorescence drop (total SV recycling pool), allowing comparison across 
A
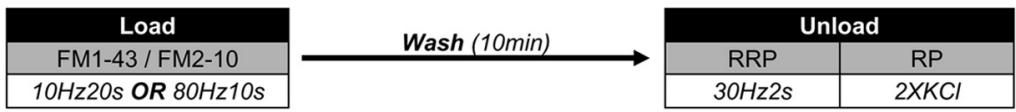

B

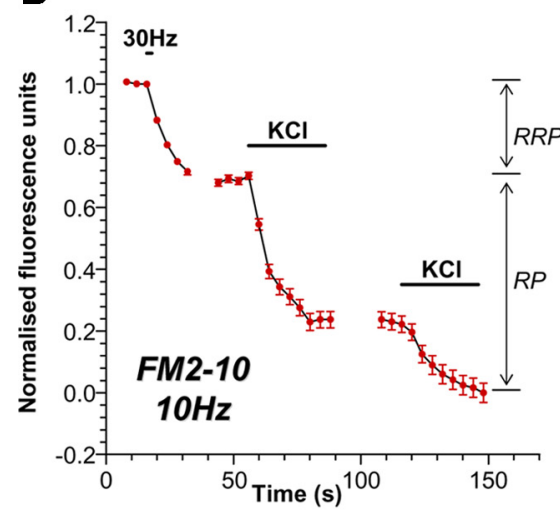

C

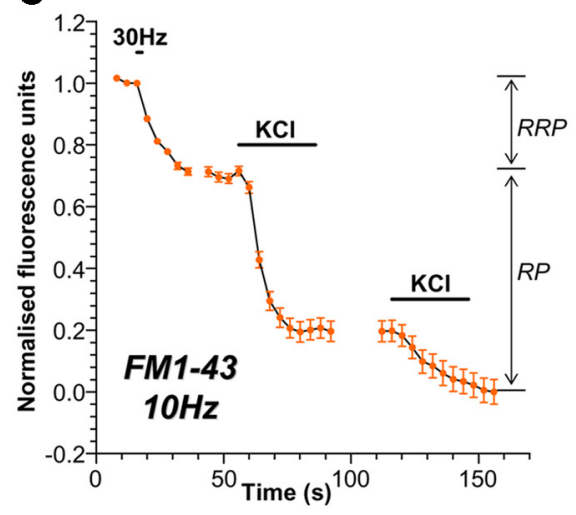

E

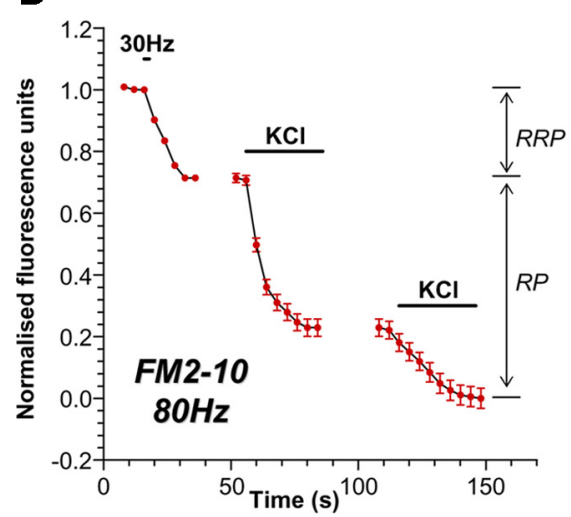

$\mathbf{F}$

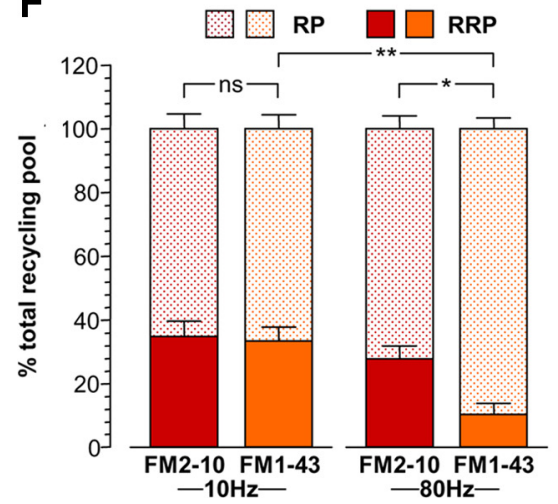

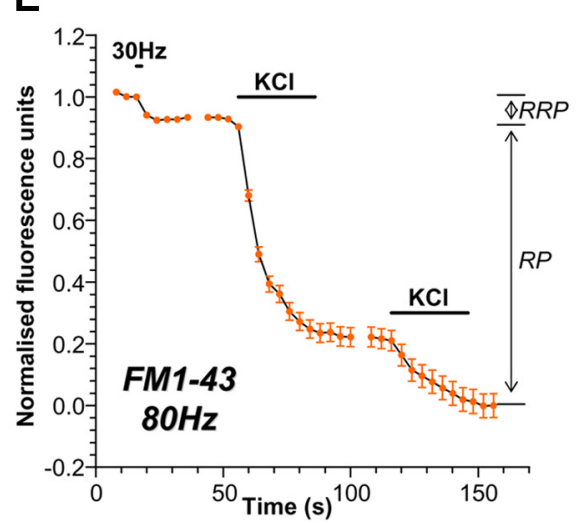

G
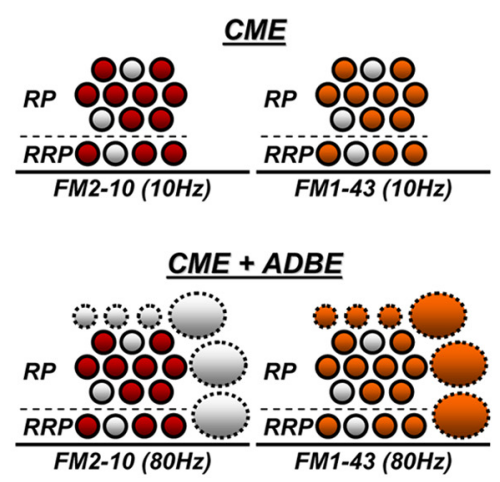

multiple experiments. For S1/S2 experiments, the S2 fluorescence drops in individual nerve terminals were expressed as a ratio of their paired internal control (S1). The data from individual nerve terminals were then averaged and these values were averaged for experiments using the same conditions. With transfected cultures, at least five nerve terminals were selected from each of the transfected and untransfected neurons in the same field of view. For direct comparison, the relative size of the reserve pool of the transfected neuron was normalized to that of the untransfected neurons in the same experiment. All calculations and statistical analyses were performed using Microsoft Excel and GraphPad Prism software.

Labeling of endocytic pathways by HRP. Cultures were fixed and processed for electron microscopy as described previously (Deák et al., 2004; Evans and Cousin, 2007). Briefly, cultures were repolarized in incubation medium for $10 \mathrm{~min}$ and stimulated with trains of action potentials in the presence of HRP $(10 \mathrm{mg} / \mathrm{ml})$. After HRP washout, cultures were either immediately fixed or challenged with further stimulation protocols in the absence of HRP (as indicated in Results) followed by fixation. In all cases, cultures were fixed in $2 \%$ glutaraldehyde for $30 \mathrm{~min}$ at $37^{\circ} \mathrm{C}$. After washing with $100 \mathrm{~mm}$ Tris, $\mathrm{pH} 7.4$, cultures were exposed to $0.1 \%$ diaminobenzidine and $0.2 \% \mathrm{H}_{2} \mathrm{O}_{2}$ in 100 $\mathrm{mm}$ Tris until color developed. Cultures were then washed with $100 \mathrm{~mm}$ Tris and stained with $1 \%$ osmium tetroxide for $30 \mathrm{~min}$. After washing in water, cultures were poststained with $2 \%$ uranyl acetate for $15 \mathrm{~min}$ and then dehydrated using ethanol series and polypropylene oxide and embedded using Durcupan. Samples were sectioned, mounted on grids, and viewed using an FEI Tecnai 12 transmission electron microscope. All nerve terminals were analyzed, and intracellular structures that were $<100 \mathrm{~nm}$ in diameter were arbitrarily designated to be SVs, whereas larger structures were considered endosomes. From these images, the number of HRP-labeled SVs and endosomes were counted in individual nerve terminals. Endosome diameter was calculated by averaging the longest and shortest diameters of individual endosomes (measured using ImageJ software, National Institutes of Health).

\section{Results}

\section{ADBE provides additional SVs to} replenish the SV recycling pool

Multiple SV retrieval modes have been identified in mammalian central nerve terminals (Royle and Lagnado, 2003; Rizzoli and Betz, 2005; Kavalali, 2006; Wu et al., 2007); however, the physiological consequences of their activation have thus far been poorly characterized. For example, the impact of the activation of different SV retrieval modes on replenishment of both the SV recycling pool and the pools within it (RRP and reserve pool) is not clearly defined. 


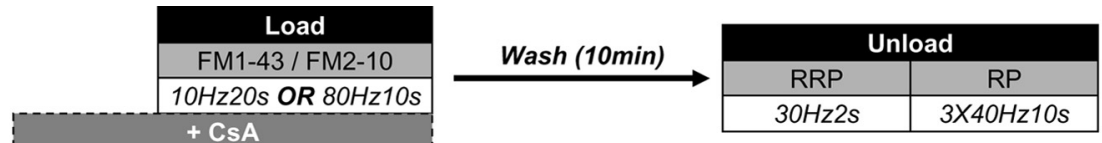

B

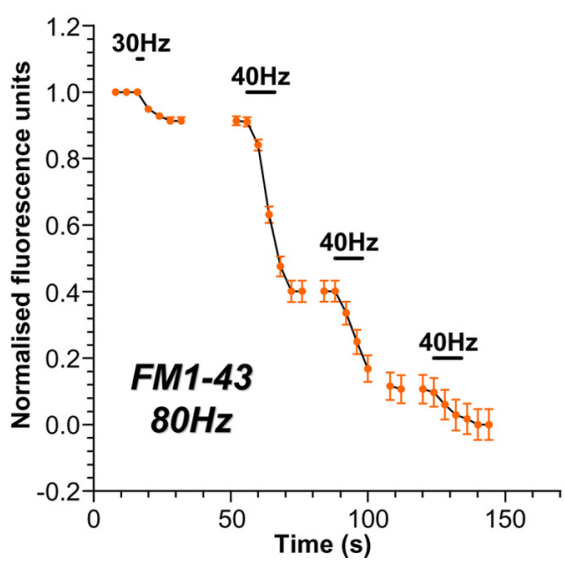

C

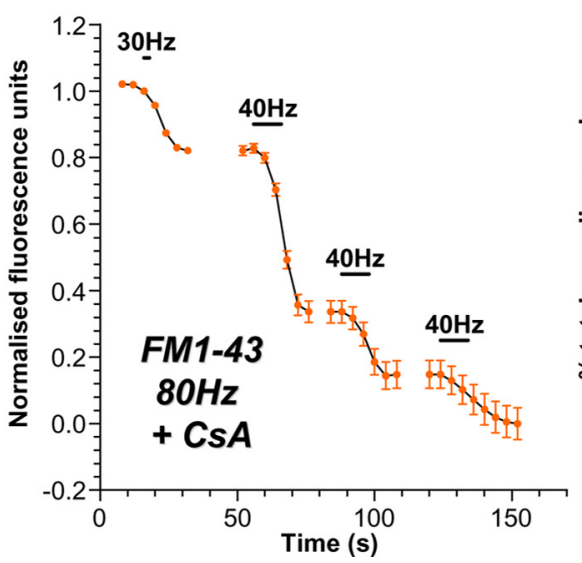

D

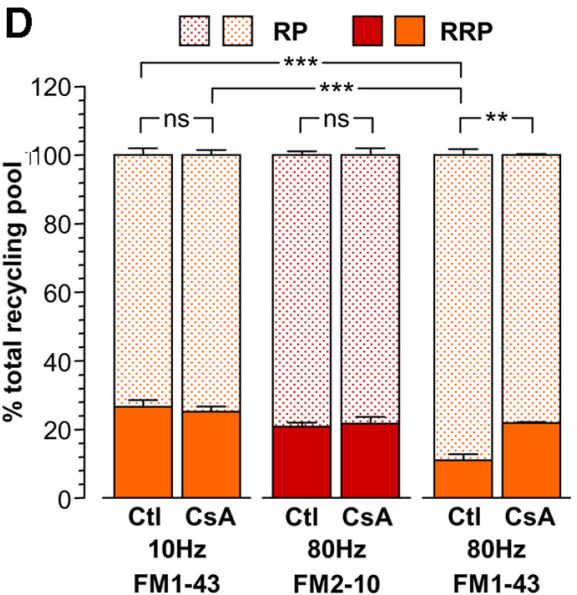

Figure 3. Replenishment of the reserve pool by ADBE is dependent on calcineurin activity. $A$, Cultures were loaded with either $10 \mu \mathrm{m}$ FM1-43 or $100 \mu \mathrm{m}$ FM2-10 using trains of either $200 \mathrm{action}$ potentials $(10 \mathrm{~Hz})$ or 800 action potentials $(80 \mathrm{~Hz})$. Dyes were washed away immediately after stimulation and cultures were left to rest for $10 \mathrm{~min}$. The RRP was unloaded with 60 action potentials $(30 \mathrm{~Hz}$ ) and the reserve pool (RP) was unloaded with three consecutive trains of 400 action potentials ( $40 \mathrm{~Hz})$. In some experiments, cyclosporin A (CsA, $10 \mu \mathrm{m}$ ) was preincubated with cultures for 10 min before and during dye loading. $\boldsymbol{B}, \boldsymbol{C}$, Representative traces of the average fluorescence drop of nerve terminals loaded with FM1-43 evoked by 800 action potentials in the absence (Ctl) or presence of $C S A$ are shown $(\boldsymbol{B}$, minus $C S A$; $\boldsymbol{C}$, plus $C S A)$. Unloading stimulations are represented by bars. Traces were normalized (between 1 and 0$)$ to the size of the recycling pool (RRP + RP) for each nerve terminal. $D$, Average proportion of the RRP and RP as a percentage of the SV recycling pool is shown (FM2-10, red bars; FM1-43, orange bars; RRP, solid bars; RP, dotted bars). In all experiments, $n=3$ (except $10 \mathrm{~Hz}$ FM1-43 control, $n=4$ ); error bars represent \pm SEM; ${ }^{* *} p<0.01$, ${ }^{* * *} p<0.001$, for both RRP and RP, one-way ANOVA.

To address this question, we first determined how the activation of either ADBE or CME impacted on the size of the subsequent SV recycling pool. This was achieved by triggering both SV retrieval modes with a maximal stimulus of elevated $\mathrm{KCl}(50$ $\mathrm{mM})$. Both CME and ADBE were labeled using FM1-43, whereas CME was labeled using FM2-10 (Evans and Cousin, 2007; Clayton and Cousin, 2008; Clayton et al., 2008, 2009). The replenishment of the total SV recycling pool was estimated by delivering trains of action potentials of increasing duration $(40 \mathrm{~Hz}$ for $10,20,40$ or $80 \mathrm{~s}$ ), as shown in Figure $1 \mathrm{~A}$. This approach allows a quantification of the time taken to exhaust the labeled pool of SVs generated by each SV retrieval mode. SVs that were generated via CME (i.e., labeled by FM2-10) were depleted after $\sim 20$ s of continual stimulation, with no additional unloading observed during longer stimulation trains (Fig. $1 B$ ). However, FM1-43-loaded SVs (generated by both CME and ADBE) continued to fuse even throughout the longest unloading stimuli (Fig. $1 B$ ). Thus, there is a clear distinction in the ability of dye-loaded SVs to contribute toward the SV recycling pool during prolonged stimulation (Fig. 1C). These experiments suggest that ABDE provides additional SVs to replenish the recycling pool, since both dyes label CME, but only FM1-43 labels ADBE. This replenishment would allow the nerve terminal to support neurotransmitter release during periods of prolonged stimulation.

\section{ADBE preferentially replenishes the $S V$ reserve pool}

We next determined whether there was specificity in the replenishment of the pools within the recycling pool (RRP and reserve pool) by either ADBE- or CME-derived SVs. This was achieved using two independent approaches. First, trains of stimuli were delivered to cultures that either only activated CME (200 action potentials at $10 \mathrm{~Hz}$ ) or activated both CME and ADBE (800 action potentials at $80 \mathrm{~Hz}$ ) (Clayton et al., 2008). Second, neuronal cultures were loaded with either FM2-10 (which selectively labels CME) or FM1-43 (which labels both CME and ADBE) during these different stimulation protocols. The extent of subsequent RRP refilling was determined by selectively unloading this pool with a brief action potential train $(60$ action potentials at $30 \mathrm{~Hz})$ (Mozhayeva et al., 2002). This protocol selectively unloaded the RRP, since application of hypertonic sucrose $(500 \mathrm{~mm}$ ) (Pyle et al., 2000; Mozhayeva et al., 2002) evoked an identical amount of dye unloading (supplemental Fig. 1, available at www.jneurosci. org as supplemental material). The remaining SVs within the recycling pool were unloaded by two sequential stimuli with 50 mM KCl (Fig. 2A). The SVs fused by this stimulation protocol were designated the reserve pool.

The refilling of the RRP and reserve pool by CME-generated SVs was determined first. CME was selectively triggered by mild stimulation ( 200 action potentials, $10 \mathrm{~Hz}$ ), and SVs retrieved by this route were loaded with either FM2-10 or FM1-43. The proportion of the RRP labeled in comparison to the reserve pool for either FM2-10- or FM1-43-loaded SVs was constant at 35\%/ $65 \%$ (RRP/reserve pool) (Fig. $2 B, C, F)$. Thus, SVs generated via CME replenish both the RRP and reserve pool of SVs.

To determine the proportion of RRP/reserve pool labeling by SVs generated by ADBE, FM2-10 or FM1-43 was loaded using 800 action potentials $(80 \mathrm{~Hz})$. When the partitioning of FM2-10labeled SVs between pools was determined, the proportion of dye loading was similar to that observed during mild stimulation (Fig. 2D,F). Thus, even at increased stimulation intensities, CME-derived SVs replenish the RRP and reserve pool to the same extent. When the partitioning of FM1-43-loaded SVs was examined, there was a dramatic shift in the proportion of SVs between the two pools. Under these conditions, only 10\% of FM1-43labeled SVs populated the RRP and 90\% populated the reserve pool (Fig. 2E,F). This experiment suggests that SVs generated by ADBE preferentially repopulate the reserve pool of SVs, since this change in SV partitioning was only observed with FM1-43 loading during high-intensity stimulation (illustrated in Fig. 2G). 
A
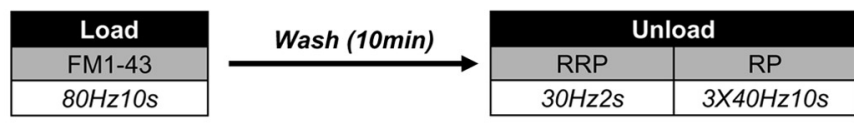

B

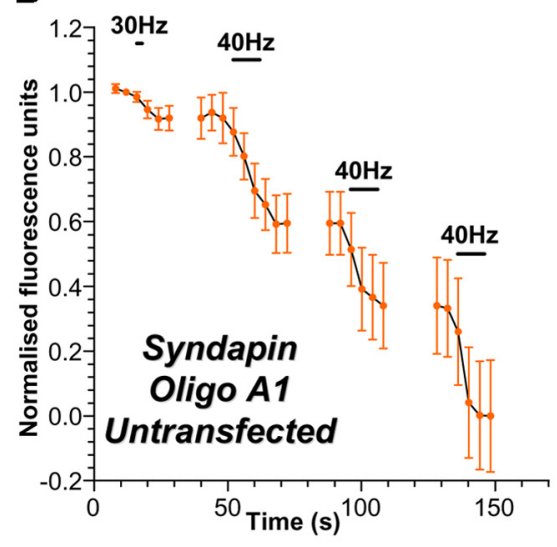

\section{C}

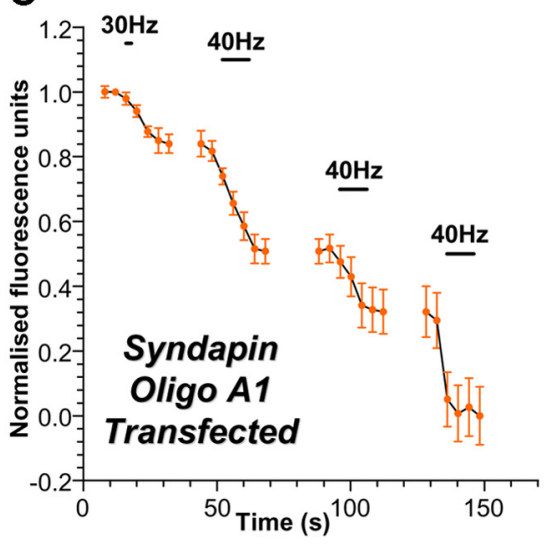

E
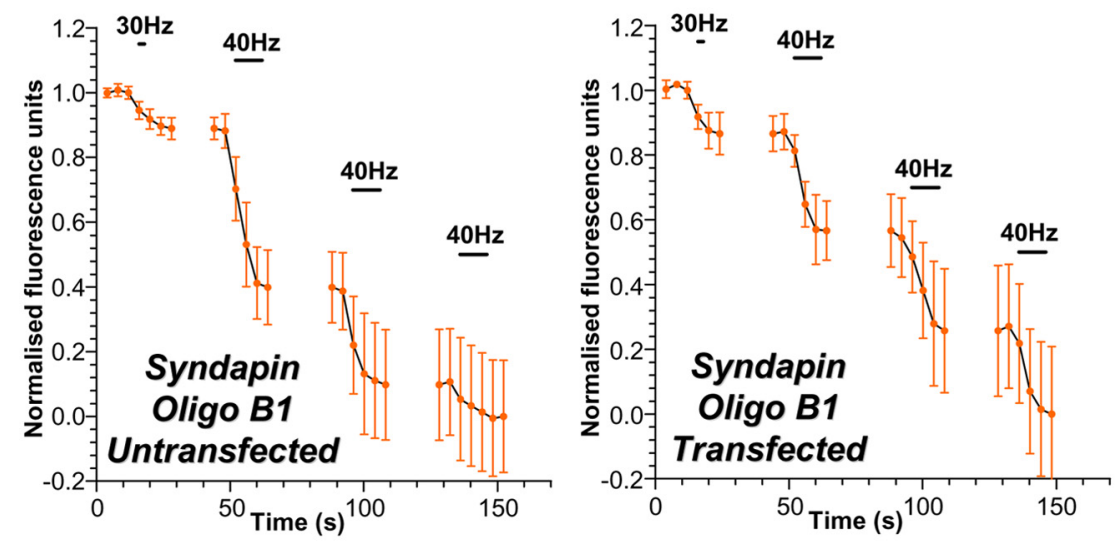

$F$

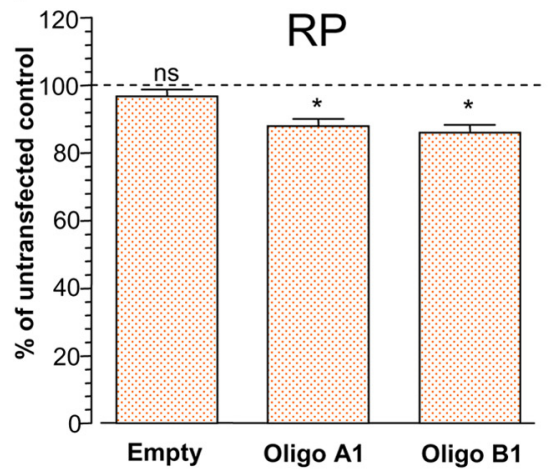

G

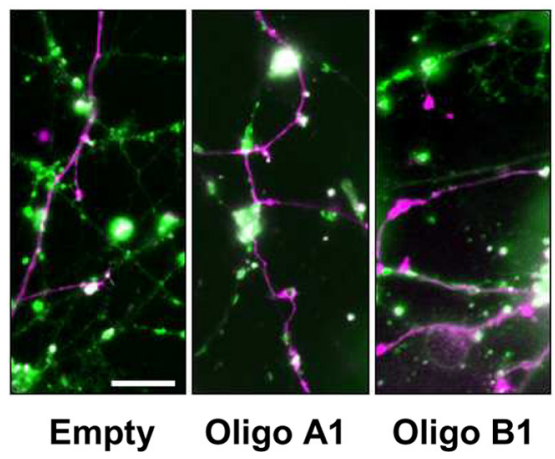

Figure 4. Replenishment of the reserve pool by ADBE is disrupted by knockdown of syndapin I expression. Cultures were transfected with either shRNA (Oligo A1, Oligo B1) against syndapin I or an empty vector $72 \mathrm{~h}$ before experiments. Transfected neurons were identified by mCerulean expression. $A$, Cultures were loaded with $10 \mu \mathrm{MFM1-43}$ using trains of 800 action potentials $(80 \mathrm{~Hz})$. Dyes were washed away immediately after stimulation and cultures were left to rest for $10 \mathrm{~min}$. The RRP was unloaded with 60 action potentials $(30 \mathrm{~Hz})$ and the reserve pool (RP) was unloaded with three consecutive trains of 400 action potentials (40 $\mathrm{Hz}$ ). $\boldsymbol{B}-\boldsymbol{E}$, Representative traces of the average fluorescence drop of FM1-43-loaded nerve terminals are displayed for cultures transfected with either Oligo $A 1(\boldsymbol{B}, \boldsymbol{C})$ or Oligo $B 1(\boldsymbol{D}, \boldsymbol{E})$ from both untransfected $(\boldsymbol{B}, \boldsymbol{D})$ and transfected neurons $(\boldsymbol{C}, \boldsymbol{E})$. Unloading stimuli are represented by bars. Traces were normalized (between 1 and 0 ) to the size of the recycling pool (RRP + RP) for each nerve terminal. $\boldsymbol{F}$, Proportions of the RP as a percentage of the SV recycling pool were determined for each nerve terminal on transfected neurons and represented as the percentage of RP unloaded from untransfected neurons in the same experiment. In all experiments, $n=3$; error bars represent \pm SEM; ${ }^{*} p<0.05$, one-sample $t$ test with proportion of RP of untransfected neurons set as 100\%. G, Fluorescent images showing FM1-43-loaded nerve terminals (FM1-43; in green) and transfected neurons (mCerulean; in magenta) for cultures transfected with empty vector (left), Oligo A1(middle), and Oligo B1 (right). Scale bar, $10 \mu \mathrm{m}$.

To confirm that the difference in partitioning of FM1-43-loaded SVs between the RRP and reserve pool was due to activation of ADBE, a series of similar experiments were performed in which nerve terminals were loaded with dye in the presence of the calcineurin antagonist cyclosporin A (Fig. $3 A$ ). Activation of the calcium-dependent protein phosphatase calcineurin triggers ADBE, and its inhibition arrests the process (Evans and Cousin, 2007). In these experiments, reserve pool unloading was evoked by three sequential trains of 400 action potentials $(40 \mathrm{~Hz})$, a protocol that is equivalent in magnitude to two challenges with $\mathrm{KCl}$ (supplemental Fig. 2, available at www. jneurosci.org as supplemental material).

Application of cyclosporin A $(10 \mu \mathrm{M})$ during FM1-43 loading evoked by mild stimulation ( 200 action potentials, $20 \mathrm{~Hz}$ ) had no effect on the partitioning of dyeloaded SVs between the RRP and reserve pool (Fig. 3D). This was also true for FM2-10 loading evoked by intense stimulation (800 action potentials) (Fig. 3D). These results were expected, since these protocols only report SVs generated by CME, a SV retrieval mode in which calcineurin has no defined role (Evans and Cousin, 2007; Clayton et al., 2008). When SVs generated by both CME and ADBE were loaded with FM1-43 during intense stimulation (800 action potentials, 80 $\mathrm{Hz}$ ), a disproportionate labeling of the reserve pool was again observed (Fig. $3 B, D)$. However, this did not occur when calcineurin was inhibited (Fig. 3C,D), with FM1-43 partitioning between pools returning to the proportions observed during either mild stimulation or with FM2-10 loading during intense stimulation (which only reports CME). Therefore SVs generated by ADBE preferentially replenish the reserve pool of SVs in central nerve terminals.

To further demonstrate a specific role for ADBE in replenishing the reserve SV pool, we determined the effect of silencing syndapin I expression on this process. Since knockdown of syndapin I inhibits ADBE (Clayton et al., 2009), this manipulation would be predicted to perturb the replenishment of the reserve SV pool. Two independent shRNA vectors containing different oligonucleotide sequences (Oligo A1 and $\mathrm{B} 1$ ) were used to silence syndapin I expression. Cultures were loaded with FM1-43 during intense stimulation (800 action potentials) (Fig. $4 A$ ) to activate ADBE. Selective unloading of both SV pools revealed that the proportion of SVs in the reserve pool was significantly 


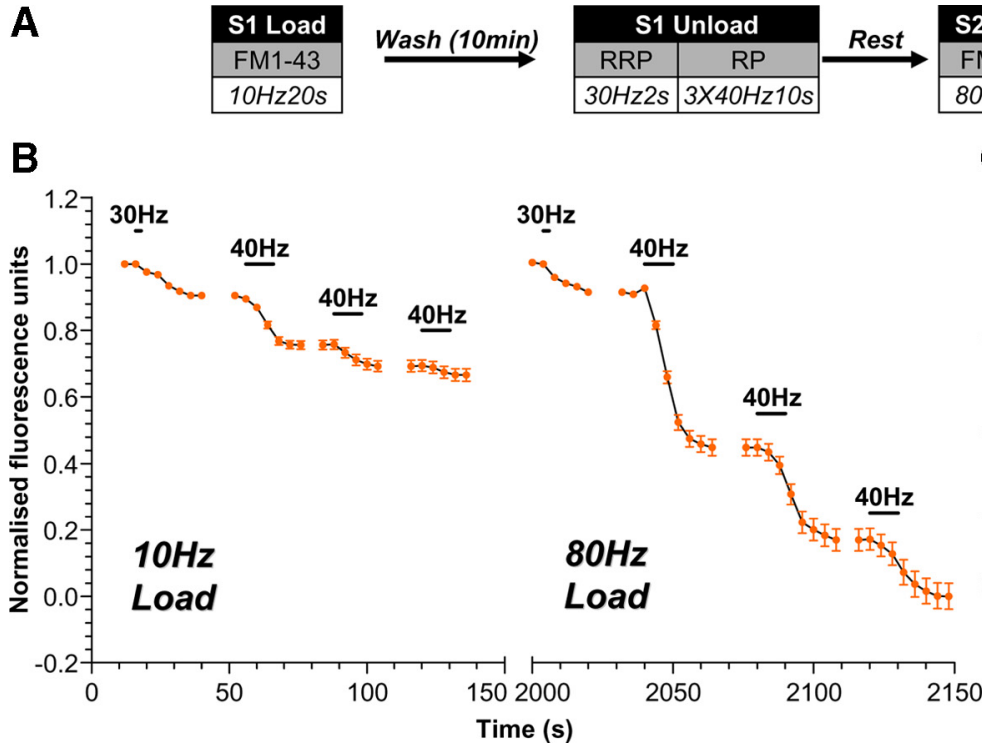

\begin{tabular}{|l|}
\hline S2 Load \\
\hline FM1-43 \\
\hline $80 H z 10 s$ \\
\hline
\end{tabular}

Wast

C

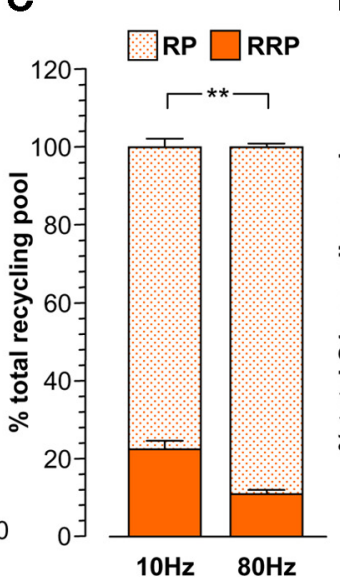

S2 Unload

\begin{tabular}{|c|c|}
\hline RRP & RP \\
\hline $30 H z 2 s$ & $3 \times 40 H z 10 s$ \\
\hline
\end{tabular}

D

Figure 5. ADBE provides additional SVs for the reserve pool but not the RRP. $A$, Cultures were loaded with $10 \mu \mathrm{MFM1}-43$ using a train of 200 action potentials ( $10 \mathrm{~Hz})$ at $S 1$ and a train of 800 action potentials $(80 \mathrm{~Hz}$ ) at $\mathrm{S} 2$. In both cases, dyes were washed away immediately after stimulation and cultures were left to rest for $10 \mathrm{~min}$. At both $\mathrm{S} 1$ and $\mathrm{S2}$, the RRP was unloaded with a train of 60 action potentials $(30 \mathrm{~Hz})$ and the reserve pool (RP) was unloaded with three consecutive trains of 400 action potentials $(40 \mathrm{~Hz})$. Cells were left to rest for 20 min between S1 and S2. B, A representative trace of the average fluorescence drop of nerve terminals is shown (10 Hz load, $\mathrm{S} 1 ; 80 \mathrm{~Hz}$ load, S2). Unloading stimulations are represented by bars. Traces were normalized (between 1 and 0 ) to the size of the 22 recycling pool (RRP + RP) for each nerve terminal. C, Proportion of the S1 and S2 RRP and RP as a percentage of their respective recycling pools is shown. $D$, Average proportion of the RRP and RP expressed as a percentage of the S1 SV recycling pool (RRP, solid bars; RP, dotted bars). In all experiments, $n=4$; error bars represent \pm SEM; ${ }^{*} p<0.05$, ${ }^{* *} p<0.01$, ${ }^{* * *} p<0.001$, for both RRP and RP, one-way ANOVA.

smaller in neurons transfected with syndapin I shRNA oligos when compared to untransfected (Fig. $4 B-F$ ). This difference was not observed in neurons transfected with the empty shRNA vector (Fig. $4 F$ ), implying a specific effect of silencing syndapin I expression in reserve pool refilling. Thus, using two manipulations that inhibit ADBE (calcineurin inhibition and syndapin I knockdown), we have shown that SVs generated via this mode selectively replenish the reserve pool.

\section{ADBE provides additional SVs for the reserve pool while the RRP is replenished by CME}

The disproportionate labeling of the reserve pool by ADBEderived SVs could arise from either provision of additional SVs for the reserve pool or a selective exclusion of SVs from the RRP. To determine the absolute size of the RRP and reserve pool that were filled by either CME- or ADBE-generated SVs, S1/S2 experiments were performed. This approach has the key advantage of comparing the effect of different stimulation protocols within the same nerve terminal.

Cultures were first loaded with FM1-43 during mild stimulation (200 action potentials, $10 \mathrm{~Hz}$ ) to evoke only CME. A similar distribution of dye-loaded SVs was observed as previously seen (Fig. 5B,C). Next, the same cultures were loaded with FM1-43 using intense stimulation ( 800 action potentials, $80 \mathrm{~Hz}$ ) to load SVs generated by both CME and ADBE. Once again, a disproportionate partitioning of FM1-43-loaded SVs into the reserve pool was observed (Fig. 5C). When the absolute amounts of dyeloaded SVs were compared between the RRP and reserve pool, key differences became apparent. First, the RRP was similar in size regardless of whether it had been labeled during either lowor high-intensity stimulation $(10 \mathrm{~Hz}, 23 \pm 2 \%$; $80 \mathrm{~Hz}, 27 \pm 5 \%$ of total S1 unload) (Fig. 5D). This indicates that the RRP must be preferentially refilled by CME-derived SVs, since no additional replenishment was observed from ADBE-derived SVs. When the number of SVs replenishing the reserve pool was compared, there was an almost threefold increase in FM1-43-loaded SVs present after intense stimulation $(10 \mathrm{~Hz}, 77 \pm 2 \%$; $80 \mathrm{~Hz}, 217 \pm 28 \%$ of total $\mathrm{S} 1$ unload) (Fig. 5D). Thus, the disproportionate labeling of the reserve pool by ADBE-derived SVs is due to a tripling in the number of releasable SVs in this pool, while the number of SVs within the RRP remains unchanged.

Parallel morphological experiments were performed examining the uptake of the fluid phase marker HRP to confirm these findings. The proportion of SVs in either the RRP or reserve pool was determined as before with the exception that cultures were left for $60 \mathrm{~min}$ before SV pool unloading to allow SV budding from bulk endosomes (Fig. 6A). First, HRP was loaded using mild stimulation (200 action potentials, $10 \mathrm{~Hz}$ ). Only HRPlabeled SVs were observed, consistent with activation of CME (Clayton et al., 2008) (Fig. 6B). This population of SVs was almost completely depleted by the RRP unloading stimulus, with very little additional unloading observed with the reserve pool stimuli (Fig. 6C,D).

Next, HRP was loaded using high-intensity stimulation (to trigger both CME and ADBE). Nerve terminals loaded with this protocol displayed a larger number of HRP-labeled SVs compared to low-intensity stimulation (Fig. 6C), agreeing with the increased number of SVs available for fusion in the SV recycling pool observed using optical imaging (Fig. 1). The RRP unloading stimulus depleted only a proportion of HRP-labeled SVs, which was almost identical in size to RRP in nerve terminals loaded with mild stimulation (Fig. 6D). This supports the hypothesis that the $\mathrm{RRP}$ is preferentially refilled by CME, since no additional SVs are present even when ADBE was active. The reserve pool stimuli unloaded an additional pool of HRP-labeled SVs that was approximately equivalent in size to the RRP (Fig. 6D). This contrasts with HRP loading by mild stimulation, where little reserve pool labeling was observed (Fig. 6D). A small population of HRP-labeled SVs did not fuse with either the RRP or reserve pool stimuli (Fig. 6D). These experiments confirm the hypothesis that 
A

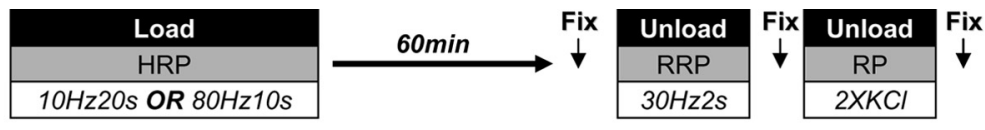

B

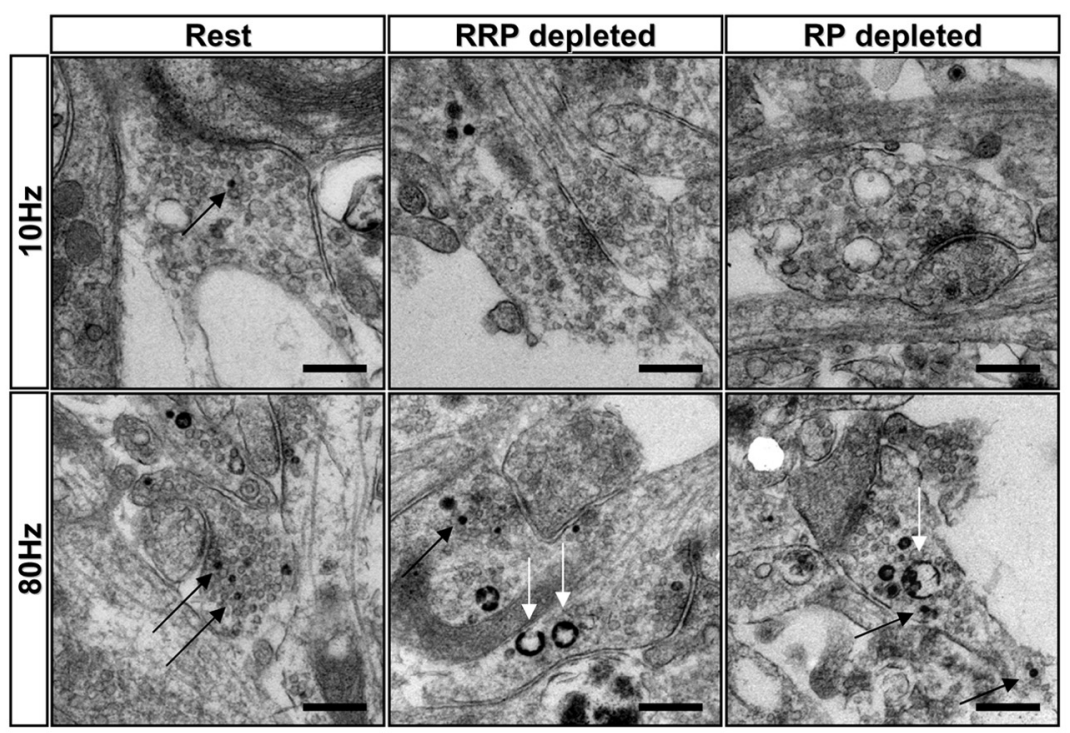

C
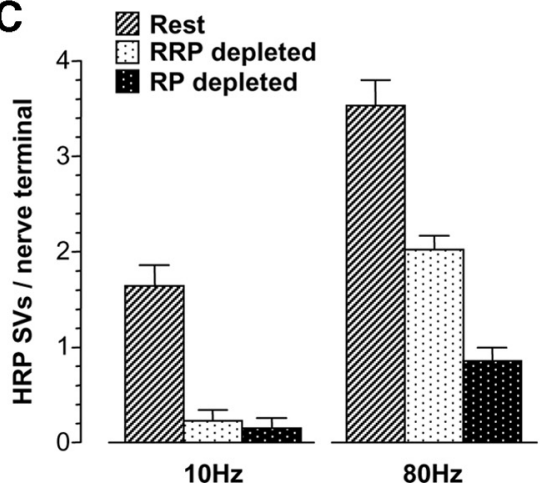

D

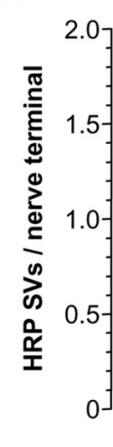

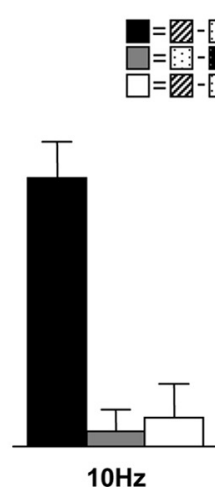

$10 \mathrm{~Hz}$

Figure 6. Replenishment of SV pools visualized by electron microscopy. $\boldsymbol{A}$, Cultures were loaded with $10 \mathrm{mg} / \mathrm{ml} \mathrm{HRP}$ using either trains of 200 action potentials $(10 \mathrm{~Hz})$ or 800 action potentials $(80 \mathrm{~Hz})$. HRP was washed away immediately after stimulation and cells were left to rest for $60 \mathrm{~min}$. The RRP was unloaded with 60 action potentials $(30 \mathrm{~Hz})$ and the reserve pool (RP) was unloaded with two 30 s applications of $50 \mathrm{~mm} \mathrm{KCl}$. Cells were fixed at three different time points indicated by arrows (rest, after RRP depletion, and after RP depletion) and processed for electron microscopy. $\boldsymbol{B}$, Representative electron micrographs display nerve terminals that were subjected to the treatments described above. Black arrows indicate HRP-labeled SVs; white arrows indicate HRP-labeled endosomes Scale bar, $500 \mathrm{~nm}$. C, HRP-labeled SVs were counted and divided by the number of nerve terminals analyzed (rest, hatched bars; RRP depleted, black dots on white bars; RP depleted, white dots on black bars). $\boldsymbol{D}$, The size of the RRP (rest minus RRP depleted), the RP (RRP depleted minus RP depleted), and an SV population belonging to neither (rest minus both RRP depleted and RP depleted) were calculated and presented as HRP-labeled SVs per nerve terminal. Data in $($ and $\boldsymbol{D}$ are averages pooled from individual nerve terminals from the same experiment ( $10 \mathrm{~Hz}$ : rest $n=56$ nerve terminals, RRP depleted $n=26$, RP depleted $n=20 ; 80 \mathrm{~Hz}$ : rest $n=75$, RRP depleted $n=112$, RP depleted $n=49$; all error bars represent \pm SEM).

ADBE provides additional SVs to preferentially replenish the reserve pool and that the RRP is preferentially refilled by CMEderived SVs.

\section{SVs generated from bulk endosomes replenish the reserve pool}

The preferential refilling of the reserve pool by ADBE can only be inferred by the previous studies, since there is no selective labeling of ADBE (FM1-43 and HRP label both ADBE and CME). To unambiguously confirm that ADBE-derived SVs preferentially refill the reserve pool, we examined pool replenishment by SVs that were specifically generated from bulk endosomes. We determined this using two separate approaches. First, we compared the absolute size of the RRP and reserve pools in the same nerve

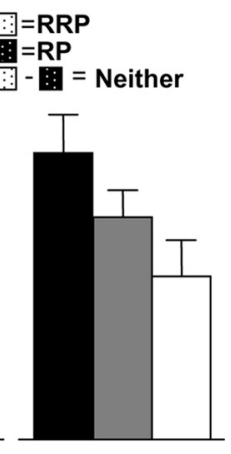

$80 \mathrm{~Hz}$ terminals either 10 min after $(\mathrm{S} 1)$ or $2 \mathrm{~min}$ after (S2) FM1-43 loading during highintensity stimulation (Fig. 7A). This protocol assumes that additional SVs that replenished pools between 2 and $10 \mathrm{~min}$ are generated from bulk endosomes. When this protocol was used, the size of RRP was constant regardless of the time period after dye loading (Fig. $7 B, C$ ). However, the reserve pool was significantly larger when cultures were rested for $10 \mathrm{~min}$ rather than 2 min after dye loading (Fig. $7 B, C$ ). Thus, FM1-43-loaded SVs that have a delayed availability for fusion replenish the reserve pool and not the RRP.

The second approach to monitor pool refilling by bulk endosome-derived SVs was to load nerve terminals with FM1-43 during high-intensity stimulation and then immediately deplete all FM1-43loaded SVs from the RRP and reserve pool (Fig. 8A). At this stage, only FM1-43loaded bulk endosomes should remain (Fig. 8 B). Cultures were then rested for 30 min to allow generation of SVs from FM143-loaded bulk endosomes (Fig. 8A, $B$ ). The refilling of the RRP and reserve pools by FM1-43-loaded SVs was then quantified by a second depletion of these pools (Fig. 8A). Immediate pool depletion revealed a larger proportion of FM1-43loaded RRP SVs than seen previously (Fig. $8 C, D)$. This was expected, since the reserve pool is replenished at a slower rate than the RRP (Fig. 7). After the $30 \mathrm{~min}$ rest period, the total SV recycling pool had $28 \pm 7 \%$ of FM1-43-loaded SVs compared to the immediate unload $(p<0.01$; one-sample $t$ test with total immediate unload set as $100 \%$ ). When dye partitioning between the RRP and reserve pool was examined for this SV population, almost all FM1-43 was in the reserve pool (90 \pm $2 \%$ of total recycling pool) (Fig. $8 D, E$ ). Thus, SVs that were generated directly from bulk endosomes specifically replenish the reserve pool.

To confirm that these newly generated SVs originate from bulk endosomes, we performed morphological analysis on cultures loaded with HRP using the same protocol (Fig. 9A). As expected, both HRP-labeled SVs and endosomes were found after HRP loading during highintensity stimulation (Fig. 9B, left). Almost all HRP-loaded SVs disappeared after immediate depletion of the RRP and reserve pool, while the same number of HRP-endosomes remained (Fig. $9 B-E)$. After the $30 \mathrm{~min}$ rest period, the number of HRP-labeled SVs increased to above the levels originally observed after HRP loading (Fig. 9B-E). Over the same time period, there was a decrease in the size of individual HRP-loaded endosomes (mean diameter $247 \pm 9 \mathrm{~nm}$ after immediate depletion; $197 \pm 5 \mathrm{~nm}$ after the $30 \mathrm{~min}$ rest period). To illustrate this, the frequency distributions of HRP-endosome diameters are plotted in Figure 9, $F$ and $G$. Thus, these results confirm that the slow replenish- 

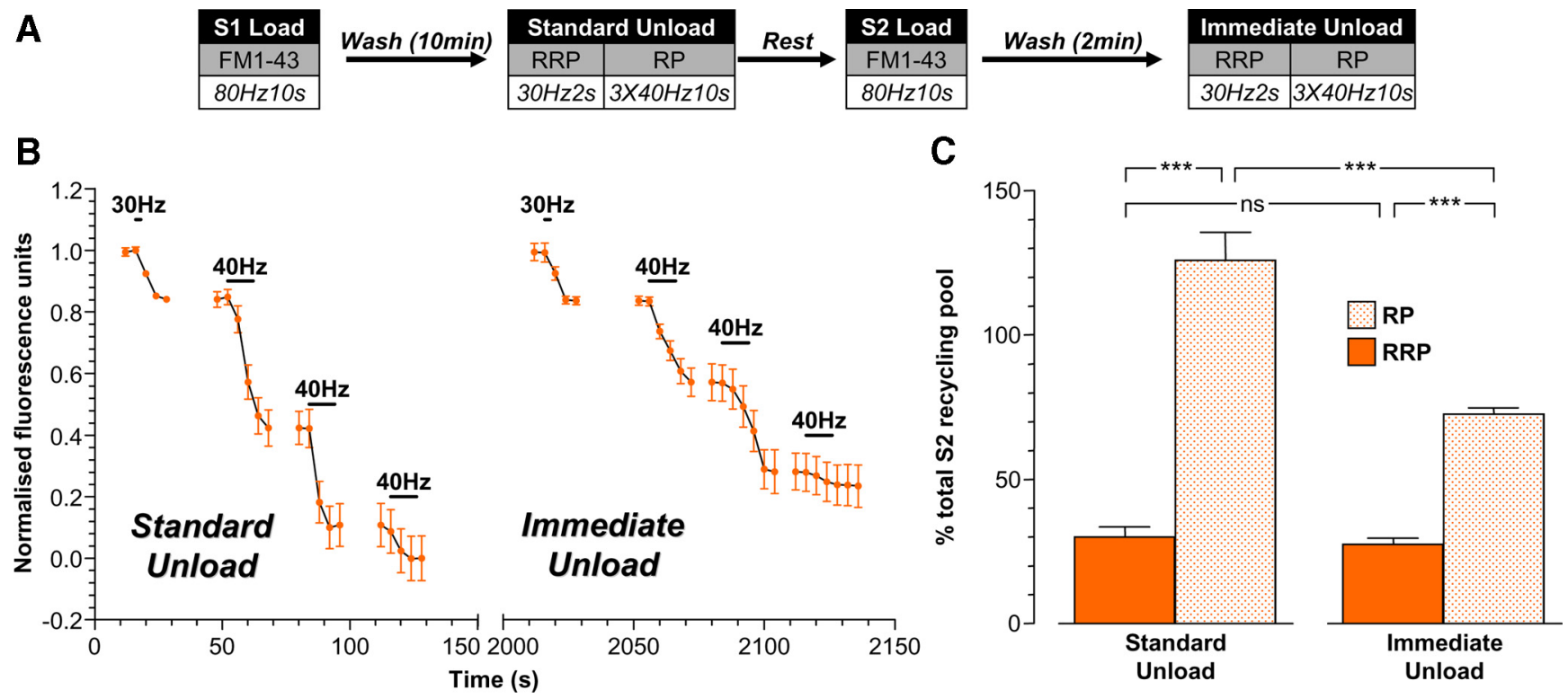

Figure 7. FM1-43-labeled SVs with delayed availability for release preferentially replenish the reserve pool. $A$, Cultures were loaded with $10 \mu \mathrm{M}$ FM1-43 using a train of 800 action potentials ( 80 $\mathrm{Hz}$ ) at both S1 and S2. In both cases, dyes were washed away immediately after stimulation and cultures were left to rest for either 10 min (standard unload) or 2 min (immediate unload) before unloading. At both S1 and S2, the RRP was unloaded with a train of 60 action potentials ( $30 \mathrm{~Hz}$ ), and the reserve pool (RP) was unloaded with three consecutive trains of 400 action potentials ( 40 $\mathrm{Hz}$ ). Cells were left to rest for 20 min between S1 and S2. B, A representative trace of the average fluorescence drop of nerve terminals is shown (standard unload, S1; immediate unload, S2). Unloading stimulations are represented by bars. Traces were normalized (between 1 and 0 ) to the size of the $S 2$ recycling pool (RRP + RP) for each nerve terminal. $C$, Average proportion of the RRP and RP in both S1 and S2 expressed as a percentage of the S2 SV recycling pool (RRP, solid bars; RP, dotted bars). In all experiments, $n=3$; error bars represent \pm SEM; ${ }^{*} p<0.05,{ }^{* *} p<0.01$, *** $p<0.001$, one-way ANOVA.

ment of the reserve pool is mediated by SVs generated from bulk endosomes.

\section{Discussion}

We have demonstrated that different SV retrieval modes are responsible for refilling specific SV pools in mammalian central nerve terminals. This was achieved by evoking either $\mathrm{CME}$ alone or $\mathrm{CME}$ and $\mathrm{ADBE}$ together and visualizing the SVs generated via these routes using selective loading of either FM1-43, FM2-10, or HRP. These studies revealed that ADBE provides additional SVs to replenish the reserve pool, whereas the RRP was preferentially refilled by CME.

The fluorescent dyes FM1-43 and FM2-10 were used to monitor both CME and ADBE retrieval modes. Previous work in both amphibian neuromuscular junctions (Richards et al., 2000) and central nerve terminals (Clayton and Cousin, 2008; Clayton et al., 2008) has shown that FM2-10 selectively labels CME, while FM1-43 labels both modes. A definitive reason for this disparity of labeling is still unclear; however, in central nerve terminals at least it seems to arise from a different affinity of the dyes for membrane being retrieved by the two SV retrieval modes (Clayton and Cousin, 2008). We are confident that we have selectively labeled SVs generated by either retrieval mode, since we used a stimulation protocol that only activated CME in our culture system (Evans and Cousin, 2007; Clayton et al., 2008; Clayton et al., 2009). During these stimulation conditions, we saw an identical pattern of loading of FM2-10 and FM1-43, suggesting that they label identical populations of SVs (CME). Furthermore, during intense stimulation (both CME and ADBE triggered), we saw an identical pattern of FM2-10 loading when compared to lowintensity stimulation, proving that this dye only labels CME. This was expected, since FM2-10 loading evoked by high-intensity stimulation is unaffected by inhibition of ADBE (Evans and Cousin, 2007; Clayton and Cousin, 2008; Clayton et al., 2009). In contrast to FM2-10, a different pattern of FM1-43 loading was observed during high-intensity stimulation, resulting in a preferential replenishment of the reserve pool. This replenishment was due to $\mathrm{ADBE}$, since it continued for up to $30 \mathrm{~min}$ after dye washout (indicating loaded SVs originated from FM1-43-loaded bulk endosomes).

The results obtained with fluorescent dyes were corroborated by morphological analysis of the uptake of the fluid phase marker HRP. In addition to confirming the dye labeling experiments, the morphological experiments provided a number of interesting findings. One surprise was that there was a population of HRPloaded SVs that were still present after unloading both the RRP and reserve pool. These might either be the remnants of bulk endosomes that are destined for degradation, or SVs that are not yet competent for release. Alternatively, these SVs may represent a replenishment of an SV pool outside the recycling pool. For instance, these HRP-loaded SV may replenish the "reluctant" SV pool, which has a lower probability of release than the RRP but can be fused by stimuli such as elevated $\mathrm{KCl}$ or increased extracellular calcium (Moulder and Mennerick, 2005). It is unlikely that these remaining HRP-loaded SV form part of such a pool, however, since we stimulated with $\mathrm{KCl}$ in our morphological experiments. What is more likely is that they have integrated into a "resting" pool of SVs. The resting pool resides outside the functional recycling pool of SVs and is not mobilized during nerve terminal stimulation (Fernandez-Alfonso and Ryan, 2008; Fredj and Burrone, 2009). In our morphological experiments, we routinely observe a loss of SVs of 35\% immediately after depletion of the recycling pool during intense stimulation [Clayton et al. (2008) and M. A. Cousin, unpublished observations], suggesting the presence of a large resting pool of SVs in our culture system. SVs can exchange between resting and recycling pools over time (Fernandez-Alfonso and Ryan, 2008). Thus, during the $60 \mathrm{~min}$ 


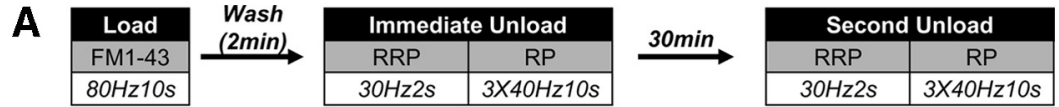

B
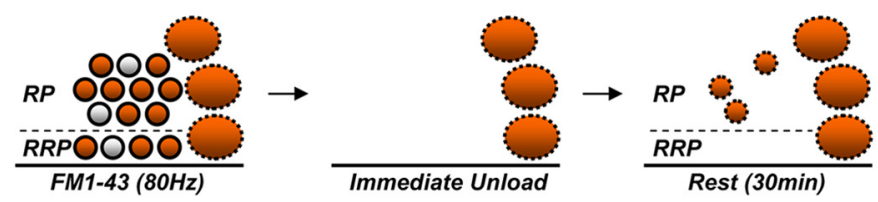

C
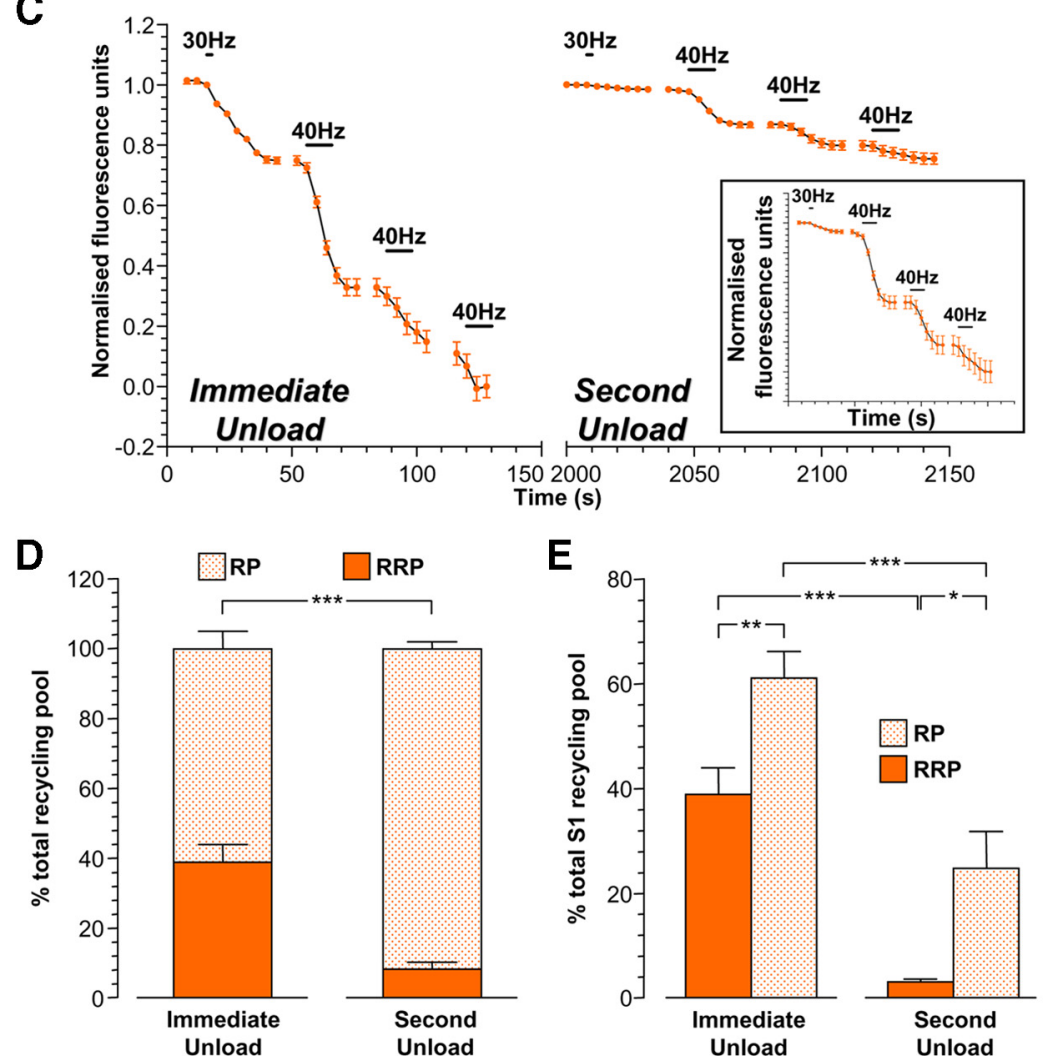

Figure 8. SVs generated from bulk endosomes specifically replenish the reserve pool. $\boldsymbol{A}$, Cultures were loaded with $10 \mu \mathrm{M}$ FM1-43 using a train of 800 action potentials $(80 \mathrm{~Hz})$. Dye was washed away immediately after stimulation and cultures were stimulated to unload both the RRP and reserve pool (RP) after both 2 min (immediate unload) and a further 30 min (second unload). The RRP was unloaded with a train of 60 action potentials $(30 \mathrm{~Hz})$ and the RP was unloaded with three consecutive trains of 400 action potentials $(40 \mathrm{~Hz})$. B, Schematic diagram illustrating how the RRP and RP are replenished by SVs loaded with FM1-43. Solid circles represent dye-loaded SVs; clear circles represent nonloaded SVs. Solid ovals represent dye-loaded endosomes. Solid outlines represent CME-generated structures; dotted outlines represent ADBE-generated structures. $C$, A representative trace of the average fluorescence drop of nerve terminals is shown (immediate unload, S1; second unload, S2). Unloading stimulations are represented by bars. Traces were normalized (between 1 and 0 ) to the size of the $S 1$ recycling pool (RRP $+R P$ ) for each nerve terminal. The inset displays the $S 2$ unload normalized to the size of the $S 2$ recycling pool. D, Proportion of the S1 and S2 RRP and RP as a percentage of their respective recycling pools is shown. $\boldsymbol{E}$, Average proportion of the RRP and RP in both S1 and S2 expressed as a percentage of the S1 SV recycling pool (RRP, solid bars; RP, dotted bars). In all experiments $n=4$; error bars represent $\pm S E M$; ${ }^{*} p<0.05,{ }^{* *} p<0.01,{ }^{* * *} p<0.001$, for both RRP and RP, one-way ANOVA.

time period before unloading the SVs generated by ADBE, there may have been exchange between these pools, resulting in some HRP-loaded SVs remaining after stimulation. The resting pool is proposed to be mobilized by spontaneous activity (Sara et al., 2005; Fredj and Burrone, 2009), and it will be of interest to determine whether these conditions evoke the remaining unloading of HRP-loaded SVs.

We are confident that bulk endosomes are the primary source of FM1-43-loaded SVs that replenish the reserve pool over time. This is because we observed a decrease in HRP-labeled bulk endosome diameter over time concomitant with an increase in the number of HRP-labeled SVs. One surprise was the longevity of HRP-labeled bulk endosomes in nerve terminals, with no obvious decrease in number observed after $30 \mathrm{~min}$. Indeed, we found HRPlabeled bulk endosomes still present in nerve terminals up to 60 min after stimulation (Fig. 6). This suggests that bulk endosomes may be able to supply SV for the reserve pool up to 60 min after stimulation has ceased. Bearing this point in mind, it will be critical to determine the molecular events responsible for the SV budding reaction from these endosomes, since this will have large implications on future reserve pool capacity.

The quantitative measurements of RRP and reserve pool capacity revealed that SVs generated by CME replenished both the RRP and reserve pool in the proportions of 30\%:70\%. Interestingly, the extent of replenishment of either the RRP or reserve pool by CME-generated SVs was not affected by increasing the intensity of the loading stimulus. This is most likely due to the fact that CME has a fixed maximal rate and limited capacity (Sankaranarayanan and Ryan, 2000; Clayton et al., 2008). Thus, once CME reaches saturation, no additional SVs can be retrieved to replenish SV pools during a fixed time period. The refilling of the RRP by CME in this study has close parallels to the mechanism of "reuse" (Pyle et al., 2000; Sara et al., 2002; Kavalali, 2006). It is still unclear what the molecular mechanism of reuse is, since reuse kinetics are faster than those published for CME of single SVs (Atluri and Ryan, 2006; Granseth et al., 2006; Kavalali, 2006; Balaji and Ryan, 2007). However recent studies have demonstrated a clathrin-dependent SV retrieval mode with much faster kinetics (Zhu et al., 2009), which suggests that the reuse mode could be a form of CME. In support, CME-generated SVs partially refilled the RRP immediately after stimuli that selectively unloaded the RRP (Granseth and Lagnado, 2008). However, at this stage, it is impossible to determine whether reuse and $\mathrm{CME}$ are the same, related, or different SV retrieval modes.

SVs generated by ADBE specifically increased the size of the reserve pool, but not the RRP. This is the first demonstration that ADBE replenishes a specific SV pool in central nerve terminals. It is also supported by previous studies in amphibian neuromuscular junctions, where SVs generated by ADBE only became available for fusion after a delayed time period (Richards et al., 2000). The selective replenishment of the reserve pool by ADBE may at first glance appear to be an obvious consequence of the fact that CME-derived SVs have already refilled the RRP. However, this may not have been the case, since in large secretory cells, for example, vesicles generated directly from the Golgi apparatus 
selectively replenish the RRP, displacing existing vesicles from this pool (Duncan et al., 2003).

This work highlights the importance of previous neuronal activity with regard to the subsequent capability of the nerve terminal to respond to stimuli. Since intense neuronal activity both mobilizes reserve pool SVs and triggers ADBE, the functional capacity of the reserve pool will be a continual equilibrium between depletion via neuronal activity and replenishment by ADBE-generated SVs. It will be of great interest to determine the impact on the capacity of the reserve pool by both the frequency and intensity of neuronal activity. For this to be determined, the whole reserve pool (i.e., existing and newly generated SVs) must be monitored, and experiments are currently in progress in our laboratory to determine this relationship.

In summary, we have shown that SVs generated via different SV retrieval modes replenish specific SV pools in mammalian nerve terminals. SVs generated by CME are responsible for the refilling of the RRP, whereas SVs generated by ADBE preferentially repopulate the reserve pool. The replenishment of these SV pools provides a key insight into how the extent of neurotransmitter release is shaped by previously experienced neuronal activity.

\section{References}

Atluri PP, Ryan TA (2006) The kinetics of synaptic vesicle reacidification at hippocampal nerve terminals. J Neurosci 26:2313-2320.

Balaji J, Ryan TA (2007) Single-vesicle imaging reveals that synaptic vesicle exocytosis and endocytosis are coupled by a single stochastic mode. Proc Natl Acad Sci U S A 104:20576-20581.

Clayton EL, Cousin MA (2008) Differential labelling of bulk endocytosis in nerve terminals by FM dyes. Neurochem Int 53:51-55.

Clayton EL, Cousin MA (2009) The molecular physiology of activity-dependent bulk endocytosis of synaptic vesicles. J Neurochem 111:901-914.

Clayton EL, Evans GJ, Cousin MA (2008) Bulk synaptic vesicle endocytosis is rapidly triggered during strong stimulation. J Neurosci 28:6627-6632.

Clayton EL, Anggono V, Smillie KJ, Chau N, Robinson PJ, Cousin MA (2009) The phosphodependent dynamin-syndapin interaction triggers activity-dependent bulk endocytosis of synaptic vesicles. J Neurosci 29:7706-7717.

Deák F, Schoch S, Liu X, Südhof TC, Kavalali ET (2004) Synaptobrevin is essential for fast synaptic-vesicle endocytosis. Nat Cell Biol 6:1102-1108

Doherty GJ, McMahon HT (2009) Mechanisms of endocytosis. Annu Rev Biochem 78:857-902.

Duncan RR, Greaves J, Wiegand UK, Matskevich I, Bodammer G, Apps DK, Shipston MJ, Chow RH (2003) Functional and spatial segregation of secretory vesicle pools according to vesicle age. Nature 422:176-180. considered endosomes.
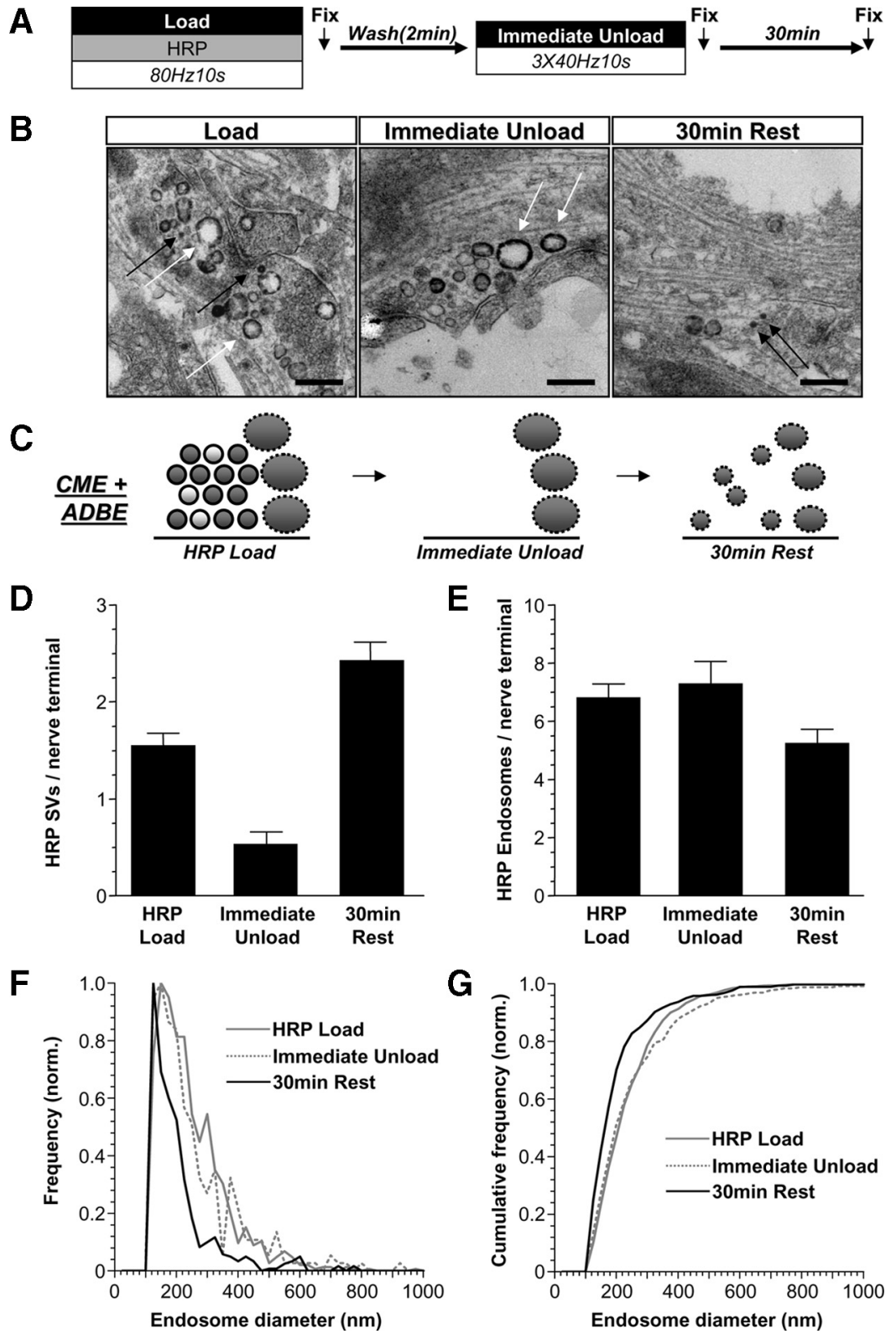

Figure 9. SVs with delayed availability for release are derived from bulk and endosomes. $\boldsymbol{A}$, Cultures were loaded with HRP (10 $\mathrm{mg} / \mathrm{ml}$ ) using a train of 800 action potentials $(80 \mathrm{~Hz}$ ). HRP was washed away immediately after stimulation, and after 2 min, cultures were stimulated with three consecutive trains of 400 action potentials $(40 \mathrm{~Hz})$ to release all available SVs. Cultures were then allowed to rest for $30 \mathrm{~min}$. Cultures were fixed at three time points indicated by arrows and processed for electron microscopy. $\boldsymbol{B}$, Representative electron micrographs display nerve terminals that were subjected to the treatments described above. Black arrows indicate HRP-labeled SVs; white arrows indicate HRP-labeled endosomes. Scale bar, $500 \mathrm{~nm}$. C, Schematic diagrams illustrating nerve terminals either immediately after HRP loading (HRP load), after depletion of all available SVs (immediate unload), or after 30 min rest. Solid circles represent HRP-loaded SVs, clear circles represent nonloaded SVs. Solid ovals represent HRP-loaded endosomes. Solid outlines represent CME-generated structures, dotted outlines represent ADBE-generated structures. $\boldsymbol{D}, \boldsymbol{E}$, Mean number of HRP-labeled SVs $(\boldsymbol{D})$ or endosomes $(\boldsymbol{E})$ per nerve terminal is displayed for each point (data pooled from 2 independent experiments; HRP load $n=137$ nerve terminals, immediate unload $n=36,30$ min rest $n=90$; all error bars represent \pm SEM). $\boldsymbol{F}, \mathbf{G}$, Endosome diameter was normalized to the total number of endosomes and plotted as frequency $(\boldsymbol{F})$ and cumulative frequency $(\boldsymbol{G})$ for each fixation time point (bin size $=25 \mathrm{~nm}$ ). Only structures with average diameters $\geq 100 \mathrm{~nm}$ were

Evans GJ, Cousin MA (2007) Activity-dependent control of slow synaptic vesicle endocytosis by cyclin-dependent kinase 5. J Neurosci 27:401-411.

Fernandez-Alfonso T, Ryan TA (2008) A heterogeneous "resting" pool of synaptic vesicles that is dynamically interchanged across boutons in mammalian CNS synapses. Brain Cell Biol 36:87-100.

Fredj NB, Burrone J (2009) A resting pool of vesicles is responsible for spontaneous vesicle fusion at the synapse. Nat Neurosci 12:751-758. 
Granseth B, Lagnado L (2008) The role of endocytosis in regulating the strength of hippocampal synapses. J Physiol 586:5969-5982.

Granseth B, Odermatt B, Royle SJ, Lagnado L (2006) Clathrin-mediated endocytosis is the dominant mechanism of vesicle retrieval at hippocampal synapses. Neuron 51:773-786.

Kavalali ET (2006) Synaptic vesicle reuse and its implications. Neuroscientist 12:57-66.

Moulder KL, Mennerick S (2005) Reluctant vesicles contribute to the total readily releasable pool in glutamatergic hippocampal neurons. J Neurosci 25:3842-3850.

Mozhayeva MG, Sara Y, Liu X, KavalaliET (2002) Development of vesicle pools during maturation of hippocampal synapses. J Neurosci 22:654-665.

Pyle JL, Kavalali ET, Piedras-Rentería ES, Tsien RW (2000) Rapid reuse of readily releasable pool vesicles at hippocampal synapses. Neuron 28:221-231.

Richards DA, Guatimosim C, Betz WJ (2000) Two endocytic recycling routes selectively fill two vesicle pools in frog motor nerve terminals. Neuron 27:551-559.

Richards DA, Rizzoli SO, Betz WJ (2004) Effects of wortmannin and latrunculin A on slow endocytosis at the frog neuromuscular junction. J Physiol 557:77-91.

Rizzoli SO, Betz WJ (2005) Synaptic vesicle pools. Nat Rev Neurosci 6:57-69.

Royle SJ, Lagnado L (2003) Endocytosis at the synaptic terminal. J Physiol 553:345-355.
Sankaranarayanan S, Ryan TA (2000) Real-time measurements of vesicleSNARE recycling in synapses of the central nervous system. Nat Cell Biol 2:197-204.

Sara Y, Mozhayeva MG, Liu X, Kavalali ET (2002) Fast vesicle recycling supports neurotransmission during sustained stimulation at hippocampal synapses. J Neurosci 22:1608-1617.

Sara Y, Virmani T, Deák F, Liu X, Kavalali ET (2005) An isolated pool of vesicles recycles at rest and drives spontaneous neurotransmission. Neuron 45:563-573.

Südhof TC (2000) The synaptic vesicle cycle revisited. Neuron 28:317-320.

Tan TC, Valova VA, Malladi CS, Graham ME, Berven LA, Jupp OJ, Hansra G, McClure SJ, Sarcevic B, Boadle RA, Larsen MR, Cousin MA, Robinson PJ (2003) Cdk5 is essential for synaptic vesicle endocytosis. Nat Cell Biol 5:701-710.

Ungewickell EJ, Hinrichsen L (2007) Endocytosis: clathrin-mediated membrane budding. Curr Opin Cell Biol 19:417-425.

Virmani T, Han W, Liu X, Südhof TC, Kavalali ET (2003) Synaptotagmin 7 splice variants differentially regulate synaptic vesicle recycling. EMBO J 22:5347-5357.

Wu LG, Ryan TA, Lagnado L (2007) Modes of vesicle retrieval at ribbon synapses, calyx-type synapses, and small central synapses. J Neurosci 27:11793-11802.

Zhu Y, Xu J, Heinemann SF (2009) Two pathways of synaptic vesicle retrieval revealed by single-vesicle imaging. Neuron 61:397-411. 